Monatsschr Kinderheilkd 2014 • 162:205-206

DOI 10.1007/s00112-013-2999-z

Online publiziert: 9. März 2014

๑) Springer-Verlag Berlin Heidelberg 2014

\author{
R. Kerbl' · P. Schober ${ }^{2}$ \\ ${ }^{1}$ Abteilung für Kinder und Jugendliche, Landeskrankenhaus Leoben \\ ${ }^{2}$ Universitätsklinik für Kinder- und Jugendchirurgie, Medizinische Universität Graz
}

\title{
Pädiatrische Sportmedizin
}

weisen sie auf die standardisierten Untersuchungsbögen der ÖGSMP (Österreichische Gesellschaft für Sportmedizin und Prävention) und empfehlen diese als allgemeines Untersuchungstool.

Das gleiche Autorenteam beschreibt in umgekehrter Autorenfolge Indikationen, Bedingungen, Ablauf und Interpretation der leistungsmedizinischen Ergometrie im Kindes- und Jugendalter. Dabei kommt meist eine sportartunspezifische Fahrrad- oder Laufbandergometrie zur Anwendung. Erstere hat gegenüber Letzterer den Vorteil der guten Standardisierbarkeit (Kurbellänge, Sattelhöhe, Lenkerstellung, Trittfrequenz), einer meist artefaktfreien EKG-Registrierung sowie relativ problemloser Blutdruckmessung und Blutabnahme zur Laktatbestimmung, sie ist außerdem auch schon bei recht jungen Kindern möglich. Ein kindergerechtes Fahrradergometer muss neben einer Verstellung der Kurbellänge sowohl eine horizontale als auch eine vertikale Veränderung von Vorbau und Sattel zulassen. Für den Ablauf der Ergometrie existieren verschiedene Protokolle. Allen gemeinsam ist, dass Ausgangsbelastung, Steigerung und Belastung in der Erholungsphase (jeweils in $\mathrm{W}$ angegeben) gewichtsangepasst gewählt werden, wobei die durchschnittliche Belastungszeit 8-12 min betragen soll. Je nach Protokoll wird alle 1-3 min auf die nächsthöhere Stufe gesteigert. Die Autoren nennen in ihrem Beitrag auch Abbruchkriterien und Kontraindikationen für die Durchführung der pädiatrischen Ergometrie. Schließlich halten sie fest, dass sich diese auch für übergewichtige und inaktive Kinder eignet und als Hilfsmittel eingesetzt werden kann, um deren Fitness und Lebensqualität zu verbessern.

\author{
》) Regelmäßige sportliche \\ Aktivität kann sich auch bei \\ chronisch kranken Kindern \\ positiv auswirken
}

K. Ruf und H. Hebestreit aus Würzburg behandeln in ihrer Arbeit das Thema der körperlichen Aktivität und Belastungsuntersuchungen bei Mukoviszidose. Sie zeigen auf, dass Kinder und Jugendliche mit dieser Erkrankung zwar verschiedene Einschränkungen haben (u. a. verminderte Atemzugvolumina, erhöhte Totraumventilation, Neigung zu Hypoxämien, Salzverlust durch Schwitzen, vermindertes Durstgefühl und damit Neigung zu Hypovolämie, Gefahr der Malnutrition, Neigung zu Hypoglykämie bei insulinbehandeltem Diabetes, Gefahr von Herzrhythmusstörungen, Osteoporose und damit Gefahr von Frakturen, Gelenkschäden), diese aber keine Kontraindikation gegen sportliche Aktivität darstellen. Im Gegenteil führt bei an Mukoviszidose erkrankten Kindern und Jugendlichen regelmäßige sportliche Aktivität zu einem Erhalt bzw. einer Verbesserung der Lungenfunktion, einer erhöhten Lebensqualität, erhöhter Leistungsfähigkeit auch bei Alltagsaktivitäten und schließlich zu einer höheren Lebenserwartung. Die Art der Sportausübung kann sich dabei durchaus an den Vorlieben der Patienten orientieren, der sog. Spaßfaktor ist für die Motivation von Bedeutung. Idealerweise wird die körperliche Leistungsfähigkeit regelmäßig durch Spiroergometrie kontrolliert, um eventuelle Komplikationen zu verhindern. Die Autoren belegen die Auswirkungen von körperlichem Training schließlich noch mit 2 Fallbeispielen. 
Dem Zusammenhang zwischen Laktatwerten und Trainingssteuerung widmet sich R. Beneke aus Marburg. Er beschreibt zunächst die Geschichte der Laktatforschung seit dessen Entdeckung im Jahr 1780 und geht in weiterer Folge auf biochemische Prozesse ein, an denen Laktat beteiligt ist. So beschreibt er u. a., dass Laktat nicht nur ein Intermediärprodukt des Kohlenhydratstoffwechsels ist, sondern auch ein Regulator der glykolytischen Flussrate und der aeroben Kohlenhydratoxidation. Der Schwerpunkt der Arbeit liegt auf der Anwendung der Laktatbestimmung für die Leistungsdiagnostik. Der Autor beschreibt in der Sportmedizin gängige Begriffe wie Laktatleistungskurve (LLK), maximales Laktat-Steady State (MLSS) und deren Beziehung zum individuellen Trainingszustand. Er verweist auf verschiedene, in der Sportmedizin (teilweise willkürlich) angewandte Schwellenwerte wie jenen bei $4 \mathrm{mmol} / \mathrm{l}$ und deren Einschränkungen sowie die Belastungsintensitätsbereiche I (Laktatwerte im Bereich des Ruhewerts oder gering höher), II (Laktatwerte deutlich höher als in Ruhe, aber im Steady State) und III (kontinuierlicher Anstieg der Laktatwerte bis zum Belastungsabbruch). Schließlich wird festgehalten, dass Laktatbestimmungen in der Phase des Aufbautrainings (ohne Maximalbelastung) weder vor noch nach der Pubertät notwendig sind, dass sie jedoch beim Höchstleistungstraining bei Jugendlichen in gleicher Weise sinnvoll sind wie bei Erwachsenen.

Schließlich wird in einem Kurzbeitrag von Schule und Kirchem auf eine Zusammenstellung der Gesellschaft für Pädiatrische Sportmedizin verwiesen, in welcher 33 Sportarten bezüglich ihrer Charakteristik, aber auch Risiken beschrieben sind. Diese Zusammenstellung findet sich unter www.kindersportmedizin.org.

Wir hoffen, mit diesem Leitthema die sog. pädiatrische Sportmedizin etwas mehr ins Bewusstsein der Kinderärzte zu bringen. Adäquate Leistungsdiagnostik dient letztlich der Sicherheit der Leistungssport betreibenden Kinder und Jugendlichen, aber auch der Absicherung jener Ärzte, die Sporttauglichkeitsuntersuchungen durchführen. Unkontrolliertes
Laufen bis zum Umfallen sollte jedenfalls der Vergangenheit angehören.

Leoben/Graz im Dezember 2013

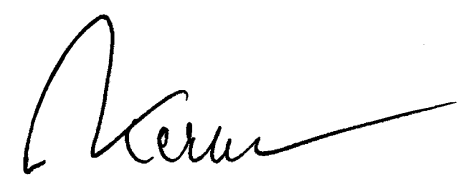

Univ. Prof. Dr. R. Kerbl

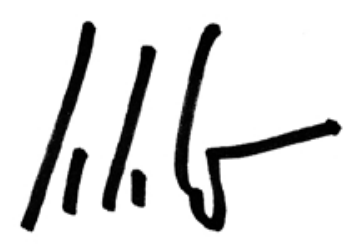

Univ. Prof. Dr. P. Schober

\section{Korrespondenzadresse}

\section{Prof. Dr. P. Schober}

Universitätsklinik für Kinder- und Jugendchirurgie, Medizinische Universität Graz Auenbruggerplatz, $8036 \mathrm{Graz}$

Österreich

Peter.schober@medunigraz.at

Interessenkonflikt. Keine Angabe.

\section{Immundefektsyndrome}

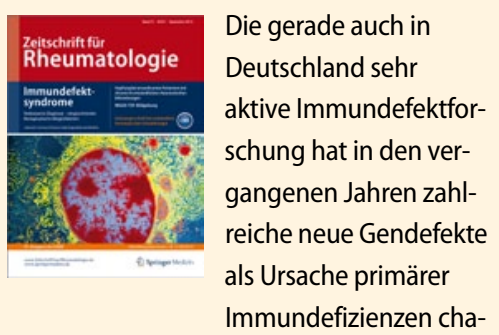

rakterisiert. Den wesentlich verbesserten diagnostischen Möglichkeiten stehen derzeit jedoch leider noch immer nur begrenzte therapeutische Möglichkeiten gegenüber. Die Beiträge der Ausgabe 7/2013 der Zeitschrift für Rheumatologie liefern einen breiten, aktuellen Überblick über Ätiologie, Pathophysiologie, Diagnostik und Therapie von primären und sekundären Immundefektsyndromen. Lesen Sie über folgende Themen ausführlicher:

- Sekundäre Immundefizienz bei rheumatologischen Erkrankungen

- Angeborene Immundefekte

- Variabler Immundefekt

- Primäre und sekundäre Neutropenie

Bestellen Sie diese Ausgabe zum Preis von 36,- Euro zzgl. Versandkosten bei Springer Customer Service Center Kundenservice Zeitschriften Haberstrasse 7

69126 Heidelberg

Tel.: +49 6221-345-4303

Fax: +49 6221-345-4229

E-Mail: leserservice@springer.com

Suchen Sie noch mehr zum Thema? Mit e.Med, dem Online-Paket von Springer Medizin, können Sie schnell und komfortabel in über 500 medizinischen Fachzeitschriften recherchieren.

Weitere Infos unter springermedizin.de/ eMed. 